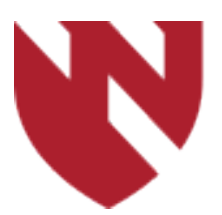

December 2019

\title{
Development of a Porcine Model of Severe Noncompressible Truncal Hemorrhage
}

\author{
Ujwal R. Yanala \\ University of Nebraska Medical Center \\ Jason Johanning \\ University of Nebraska Medical Center \\ Iraklis Pipinos \\ University of Nebraska Medical Center \\ William Velander \\ University of Nebraska Medical Center \\ Mark Carlson \\ University of Nebraska Medical Center
}

Tell us how you used this information in this short survey.

Follow this and additional works at: https://digitalcommons.unmc.edu/gmerj

Part of the Higher Education Commons, and the Medicine and Health Sciences Commons

\section{Recommended Citation}

Yanala, U. R., Johanning, J., Pipinos, I., Velander, W., , Carlson, M. Development of a Porcine Model of Severe Noncompressible Truncal Hemorrhage. Graduate Medical Education Research Journal. 2019 Dec 13; 1(1).

https://digitalcommons.unmc.edu/gmerj/vol1/iss1/47

This Conference Proceeding is brought to you for free and open access by DigitalCommons@UNMC. It has been accepted for inclusion in Graduate Medical Education Research Journal by an authorized editor of DigitalCommons@UNMC.For more information, please contact digitalcommons@unmc.edu. 


\section{Development of a Porcine Model of Severe Noncompressible Truncal}

Hemorrhage

\section{Creative Commons License}

\section{(c) (1) $\Theta(9$}

This work is licensed under a Creative Commons Attribution-Noncommercial-No Derivative Works 4.0 License. 
all treatment approaches, was 5.2\% (95\% CI $5-5.5 \%), 3.9 \%(95 \%$ CI $3.4-4.4 \%)$ and $7.8 \%$ (95\% CI 7.2-8.5\%) respectively. For patients that underwent surgery, the 5-year survival for patients with lesions in all locations, head, body and tail was $18.8 \%, 15.9 \%, 23.8 \%$ and $33.8 \%$ respectively. When analyzing trends we noted a significant improvement in survival for patients that underwent operative

\section{Global Trends in Esophageal Cancer}

Gautam K. Malhotra, Ujwal Yanal, Advaitaa Ravipati, Matthew Follet, M. Vijayakumar, Chandrakanth Are

Mentor: Chandrakanth Are

Program: General Surgery

Background \& Objectives: Esophageal Cancer (EC) is a lethal malignancy with poor prognosis and significant variations in the incidence, mortality, and histopathology based on geographic regions. The aim of this study was to quantitatively analyze these variations to identify patterns and areas for further research.

Methods: We utilized the GLOBOCAN 2012, and Cancer Incidence in five Continents, intervention. Comparing the time periods from 1977-1981 versus 2002-2006, the 5-year survival for surgical patients improved for all locations $(9.2 \%$ to $22.6 \%, \mathrm{p}<0.05)$ and also for lesions in the head $(8.3 \%$ to $19.3 \%$, $\mathrm{p}<0.05)$, body $(6.7 \%$ to $26 \%, \mathrm{p}<0.05)$ and tail ( $14.7 \%$ to $40.7 \%, \mathrm{p}<0.05)$.

Conclusion: The results of our study demonstrate an improvement in survival for patients with pancreatic cancer treated by operative intervention. This improvement in survival was more pronounced for patients with primary lesions located in the body and tail.

https://doi.org/10.32873/unmc.dc.gmerj.1.1.044
Volume X (CI5X) database to analyze variations in $\mathrm{EC}$ incidence and mortality.

Results: We found the EC incidence and mortality is geographically varied with a particularly high burden in East Asia and Eastern/Southern Africa where esophageal squamous cell carcinoma (SCC) predominates over adenocarcinoma (AC). Interestingly, there is a dichotomy between the high incidence of esophageal SCC in East Africa and low incidence in West Africa. The global incidence and mortality from $\mathrm{EC}$ is expected to rise in the coming decades. Asia, and
China in particular, will continue to be the areas most burdened by EC, while Africa is expected to surpass the incidence and mortality rates of Europe.

Conclusions: The global burden of EC is expected to rise in the coming years. Understanding the geographic, environmental, and genetic contributors to the development of EC will be essential in combating its prevalence.

https://doi.org/10.32873/unmc.dc.gmerj.1.1.045

\section{Outcomes of Intragastric Balloon Placements: An Analysis of the 2016 MBSAQIP Database \\ Bhavani Pokala, Priscila Armijo, Dmitry Oleynikov}

Mentor: Dmitry Oleynikov

Program: General Surgery

Background \& Objectives: Intragastric balloon placement is an approved weightloss procedure with a lower BMI cutoff than bariatric surgery. The objective of this study was to examine the frequency and outcomes of balloon placements in 2016.

Methods: The MBSAQIP database was analyzed for adults who underwent elective, endoscopic intragastric balloon placement. Emergent cases, patients with previous foregut surgery and those unable to be followed for 30 days were excluded. Comparisons were made between placements performed by GI and surgery. Analysis performed using SPSS v25.0, $\alpha=.05$.

Results: Our study included 445 patients. Majority were female (80\%) and Caucasian (90.6\%). Median age and BMI were 49.0 (IQR 15.4) and 35.7 (IQR 6.46), respectively. Prevalence of medically treated hypertension was $30.8 \%$, hyperlipidemia $18.9 \%$, GERD $20.4 \%$, and diabetes $12.6 \%$ (insulin and non-insulin dependent). Mean operative time was $15.48 \pm 9.89$ minutes. No 30 -day mortalities were reported. 70 balloons were placed by GI and 375 by surgery. There was a significantly higher rate of readmission for GI $7.1 \%$ vs $1.9 \%$ surgery ( $\mathrm{p}=.027)$. No significant difference was seen for rates of post-operative dehydration (GI: 7.1\% vs surgery: 7.2\%; $\mathrm{p}=1.000$ ), emergency room encounters (GI: $14.0 \%$ vs surgery: $4.0 \% ; \mathrm{p}=.486), 30$-day reoperations (GI: $0.0 \%$ vs surgery: $0.8 \%$; $\mathrm{p}=1.000$ ), and 30-day reinterventions (GI: $5.7 \%$ vs surgery $2.4 \% ; \mathrm{p}=.132$ ).

Conclusions: The majority of patients undergoing intragastric balloon placement fall within obesity class 1 or 2 and have low rates of comorbidities. The procedure can be performed by gastroenterology or surgery at bariatric centers with similar outcomes. https://doi.org/10.32873/unmc.dc.gmerj.1.1.046

\section{Development of a Porcine Model of Severe Noncompressible Truncal Hemorrhage \\ Ujwal Yanala, Jason Johanning, Iraklis Pipinos, William Velander, Mark Carlson}

\section{Mentor: Mark Carlson}

Program: General Surgery

Introduction: Noncompressible truncal hemorrhage and brain injury account for most early mortality in military personnel on the battlefield. Currently there is no effective treatment for noncompressible truncal hemorrhage, other than rapid evacuation to a surgical facility. We intended to develop a porcine model of noncompressible truncal hemorrhage so that we might be able to develop new treatments for this clinical problem.

Methods: Swine (boars, 3 months, 32-34 kg) underwent one of three injury types: 1) central stellate injury, just anterior to suprahepatic inferior vena cava $(\mathrm{N}=6) ; 2$ ) excision of a portal vein branch distal to the main trunk $(\mathrm{N}=5)$; or 3) near-transection of the left lateral lobe (LLL) of the liver at its base, which cut the hepatic and portal veins to this lobe $(\mathrm{N}=12)$. The midline incision was towel clipped immediately after injury, and animals were monitored for $60 \mathrm{~min}$ or until death. Resuscitation was performed with warm LR (max volume $=100 \mathrm{~mL} / \mathrm{kg}$ ), with a target MAP set at $80 \%$ of pre-injury MAP.

Results: The starting weight, MAP, hemoglobin, arterial $\mathrm{pH}$, INR, and pre-injury blood loss did not differ among groups $(\mathrm{p}$ 
$>0.05$, ANOVA). Postmortem evaluation revealed that 2-3 hepatic veins were transected by injury type $1,1-2$ portal vein branches plus 1 hepatic vein were transected by injury type 3 (LLL transection).

Conclusions: The hemorrhage severity of the central stellate injury was inadequate, while hemorrhage from excision of a proximal branch of the portal vein appeared to be too severe (i.e., the subjects died too quickly).
Near-transection of the hepatic LLL at its base appeared to yield hemorrhage of appropriate severity, as indicated by the $40 \% 1$-hour mortality and intermediate values for MAP, blood loss, and other variables. Our plan is to use this latter injury model in the development of therapies for noncompressible truncal hemorrhage.

https://doi.org/10.32873/unmc.dc.gmerj.1.1.047

\section{Exogenous Testosterone Supplementation as Cause of Ischemic Stroke, Mediated through Secondary Erythrocytosis \\ Fuad-al Ali, Amy Hellman, Pierre Fayad}

Mentor: Pierre Fayad

Program: Neurology

Background: The use of exogenous testosterone has become more prevalent in recent years but the effects of such use in regards to potential cerebrovascular disease have yet to come to a conclusion. In this case report, we discuss a patient who suffered an ischemic stroke due to testosterone supplementation.

Case presentation: A middle-aged patient with history of hypertension and hyperlipidemia that presented with falls, difficulty performing simple tasks and slurred speech. Patient had been taking testosterone injections every 6 months for the prior 2 years for loss of energy and reported low levels. Lab work revealed a hemoglobin of $20 \mathrm{~g} / \mathrm{dL}$ and a hematocrit of $51.5 \%$. MRI of the brain showed a left striatocapsular ischemic infarct. He was given the diagnosis of ischemic stroke due to polycythemia and thrombus formation secondary to testosterone supplementation.

Discussion: Testosterone and dihydrotestosterone (DHT) has both protective and adverse effects. Both low and high levels of testosterone and DHT have an association with ischemic stroke and there may be an optimal level that is safe.

Conclusion: The risk of ischemic stroke may be related to testosterone and DHT levels but no clear guidelines exist on optimal levels in supplementation. Thus, clinicians should be aware of the potential risk and monitor androgen and hematocrit levels.

https://doi.org/10.32873/unmc.dc.gmerj.1.1.048

\section{Pharmacological Treatment of Status Epilepticus at UNMC: Assessment of Seizure Outcomes}

Hae Young Baang, Nicholas Swingle, Kalyan Sajja, Deepak Madhavan, Olga Taraschenko

Mentor: Olga Taraschenko

Program: Neurology

Objective: To assess the effectiveness of treatment for status epilepticus (SE) at UNMC and determine patient health outcomes related to the delayed control of seizures

Design/Methods: Retrospective chart review was performed on 1565 adult patients who underwent continuous video electroencephalography (cvEEG) for suspected seizures between January 1, 2012 and February 28, 2018.

Results: 49 patients had and were treated for 64 incidences of convulsive or nonconvulsive
SE recorded on cvEEG or clinical seizures longer than $5 \mathrm{~min}$ which were documented by a neurologist. Among 64 cases, 55 incidences required the second AED treatment, and 40 out of 55 cases were treated with the third AED subsequently. The mean seizure detection-to-needle time for the first, second, and third antiepileptic drugs were 167, 239, and 297 minutes, respectively. Benzodiazepines were administered as the first AED in only $46 \%$ of all treated cases. The duration of the hospital stays in patients treated with the first AED within 60 min after seizure detection was shorter compared to those whose treatment was delayed (23.0 vs.
33.6 days, $\mathrm{p}=0.41$ ). The mortality rate in the former group was lower than that in the group which did not receive AEDs within $60 \mathrm{~min}$ (28.6 vs. $53.6 \%, \mathrm{p}=0.02$ ).

Conclusion: The current treatment approaches for SE at UNMC are inefficient with significant delay in delivery of medications after seizure detection; they may contribute to the prolonged hospital stay and increased mortality. In order to improve patient care, the existing protocol for the treatment of SE at UNMC must be revised.

https://doi.org/10.32873/unmc.dc.gmerj.1.1.049

\section{A Clinical and Cost Effectiveness Comparison of Traditional VNS Therapy to Aspire Sr (106) \\ Krishna Mourya Galla, Kalyan Sajja, Christopher Wichman, Hongmei Wang, Deepak Madhavan}

Mentor: Deepak Madhavan

Program: Neurology

Background: VNS Therapy has demonstrated overall cost savings in patients with drug- resistant epilepsy. The overall goal of this project is to evaluate the clinical and cost effectiveness of the Aspire SR 106 VNS (Vagus Nerve Stimulator) system, using retrospective patient data. We anticipate the extra dimension of closed-loop autostimulation in the Aspire 106 model will demonstrate more efficient utilization of health care resources. 